\title{
Comparing the Efficiency of Cohort, Time Homogeneous and Non-Homogeneous Techniques of Estimation in Credit Migration Matrices
}

\author{
Fred Nyamitago Monari ${ }^{1}$, Dr. George Otieno Orwa ${ }^{2}$, \\ Prof. Romanus Otieno Odhiambo ${ }^{3}$ Dr. Joseph Kyalo Mung'atu ${ }^{4}$ \\ Department of Statistics and Actuarial Science, Jomo Kenyatta \\ University of Agriculture and Technology, Kenya
}

\begin{abstract}
In many applications of Risk Management nature, Credit Migration Matrices or Transitional matrices are the main Cardinal inputs and the accuracy of their estimation is very critical. Three approaches namely Cohort, time homogeneous, non homogeneous are explored in this paper and the differences resulting statistically highlighted. Using techniques of bootstraps, a testing procedure is developed for assessing statistically the migration matrices differences. We find that using the Frequentist (Cohort) approach can yield huge discrepancies than the two duration methods namely Homogeneous and NonHomogeneous duration methods. The more efficient Duration methods when put into use will have substantial significance in Capital Credit Risk differences between recession and expansion and economic regimes. Depending on the estimation technique used, the difference can be large and damaging and can result in possibility of false assumption being made of time homogeneity.
\end{abstract}

Keywords: Risk management, Credit Migration, Credit Risk, Transitional Matrices, Bootstrap

\section{Introduction}

In many applications of risk management nature, Credit derivative pricing, term structure modeling of Credit risk and portfolio assessment of risk in Credit portfolios, Transitional Matrices or Credit migration are major inputs. Ratings of Migration drive the Capital requirements in the New Basel Accord (BIS(2001)) and there level of accuracy ib estimation is very critical. Three approaches namely Frequentist (Cohort). Time Homogeneous Duration and Non-Time Homogeneous Duration are explored in this research. And their resulting differences compared statistically, by use of eigen value, matrix norms, Vector analysis, credit portfolios and models of Credit derivatives. By making use of Bootstrap technique, a procedure of testing which is based on Singular value decomposition is used to statistically asses the differences between the Matrices of Markov nature. Economically, the method can have a huge impact since different techniques of estimations used in getting the differences in Credit Risk Capital will be as large as the differences between recession and expansion of economic regimes. We find that the Duration methods are more efficient and ignoring the two methods can result in a possibility of false assumption being made of time homogeneity, which is very significant given that the Frequentist (Cohort) method is popular with Researchers and Practitioners.

Agencies like Standard and Poor's, Moody's and Fitch produce Credit ratings which provide informed opinions to participants in the market. The ratings are important since they provide s source of information to investors and the credit market. The Credit market has grown International in recent years to include a wide range of products, obligors and structures. With the new Basel accord, new rules for ratings have been acquired as authorities doing supervisory roles have made it regulatory requirements for rating contingency. Developments in the Credit and Bond market in the recent Years have changed the techniques of portfolio management. In today's world, more resources and efforts have been put to assess Credit risk and the tools for assessing the Credit risk have become more sophisticated with the focus continuing being made on activities in the market and innovations of Finance products which are complex e.g Collateral Debt Obligation (CDO), Credit Default Swaps (CDS) etc.

Credit products move from one category of rating to another with time and emphasis has been put on understanding default risk as well as Transition risk. This means that assessment of Credit quality changes by rating agencies result in quality of obligor's Credit being revealed and this is what is known as Credit rating migration. Volatility in ratings and risk in default across the ratings spectrum are likely to change e.g. they increase with each movement consecutively in the scale of ratings downwards and in situations where an investment is moving from one grade of investment to a speculative grade. Transition rating frequencies i.e probabilities are inputs which are useful in estimating the distribution in loss as the obligors quality change and they compute measures in Var (Value at risk). 
Scenarios on Credit analysis are also prepared hence with all these reasons, in many applications of risk management, the data in Credit migration have become cardinal inputs. Transition rating frequencies which are based on historical data have specified periods of time in which they can be computed and for estimation of transition probabilities which are historical, they can be used under assumptions which are certain. For pricing financial instruments which are sensitive in terms of credit e.g. Credit default Swaps (CDS) which depend on changes in credit rating, rather than using historical transition probabilities, risk neutral is the one used. To get a Credit transitional matrix which is risk neutral, modification in historical probabilities are done in ways that prices in stock markets are matched with default probabilities. Rating transition differences are subjected to statistical tests which are rigorous and an analytical approach from the theory of random matrices is relied upon to get ratings transition spectrum confidence intervals. Ratings consistency are shown in the statistical tests i.e credit quality overall degree is compared across the products, sectors and regions. In getting rating momentum measures, ratings in structured finance and corporate are determined to see whether path dependency characteristics are exhibited i.e whether the action in rating for one Year impacts on the movement in rating of the following year. A square matrix $\mathrm{A}$ is called a transitional matrix if all entries of $\mathrm{A}$ are non-negative and the sum of entries in any given row is 1 . Probabilities of migrating from one state to another is given by each row. The use of transition matrices in modeling Credit Risk has been receiving increasing attention since Jarrow, Lando and Turnbull(1997) paper. An improvement on procedure of estimation in Jarrow et Al (1997) was undertaken by Kijima and Komoribayashi(1998. Modelling Credit transitions rating one factor Markov process was proposed by Belkin Suchower and Forest $\operatorname{Jr}(1998)$.

\subsection{Transitional Matrices Estimation}

\section{Methodology}

At a time $\mathrm{T}$, the migration or Transition of a matrix $\mathrm{P}$ is described by the probabilities of it being in any grade at time $\mathrm{T}+1$. Jarrow, Lando and Turnbull (1997) explained the difference between implicit and explicit transitionalMatrices estimation and said that estimation of Migration (Transition) Matrices from historical data where extraction of default and transitional information from the current market prices of zero coupon bonds that are risky. Various explicit articles will be considered in this article.

\subsection{Frequentist Method (Cohort approach)}

Frequentist approach over the years has been adopted as industry standard since it is straight forward. Let the probability of migrating or transitioning from $\mathrm{i}$ to $\mathrm{j}$ be $P_{i j}^{(\Delta t)}$. For example at year $\Delta \mathrm{t}=1$, at the beginning of the year, there are $N_{i}$ firms in category of rating $i$ and at the end of the year, $N_{i j}$ had migrated to grade $j$.

Transition probability estimate is given by;

$$
P_{i j}^{(\Delta t=1 \text { year })} \text { is } P_{i j}^{(1)=\frac{N_{i j}}{N_{i}}}
$$

The proportion of firms at period end $i$, year end with $j$ rating having started with $i$ rating is the probability estimate and any change occurring within the period is ignored, which is a major weakness of this method.

\subsection{Hazard rate or Duration Method}

Parallels may be drawn between histories of rating of a firm and any other event data e.g treatment trials at a clinic or histories of unemployment. In all cases, follow-ups are made as there are transitions from one state to another e.g in the case of patients, from sick state to healthy state. Two key aspects found in rating credit histories are censoring from the right where it is not known what happens to a firm when there is closure of sample window (i.e is there immediate default or no default) and left truncation where only firms have survived by enough and have been given a rating in the sample. Frequentist or Cohort method ignored both of these issues. Survival analysis attempts to address these issues. In a Markov chain which is $\mathrm{K}$-state homogeneous, state 1 indicates the highest rating i.e AAA and the worst rating is state $\mathrm{k}$ i.e default. The probability transition matrix is a distance function between dates and not the dates where you are. The methods of estimation will be dictated by relaxing or accepting the assumption of time homogeneity. Existence of a Markov transition matrix which is empirically observed was developed by Israel, Rosenthal and Wei(2001). They showed that there is no compatibility between annual Credit transition matrices with continuous Markov process mostly but not exclusively because of their sparseness and many elements which are zero in value are on far of diagonals because probability mass lies on the diagonal. They use the L norm to compare the matrices after making 
a series of adjustments. However, this method does not allow for estimation noise or sampling and it does not have a formal hypothesis test thus making it difficult to ascertain in the matrix comparison, how small or how close the matrix is from Markov compatibility.

\subsection{Case of Time Homogeneous}

Assuming time homogeneity, a $\mathrm{k} \times \mathrm{k}$ generator can describe transition matrices of matrix A.

$P(t)$ is defined as a $k \times k$ matrix of probabilities where element $i j$ is the migratory probability of moving from state $i$ to state $j$ in a period of time $t$.

$$
P(t)=\exp (\Lambda t) t \geq 0
$$

where the matrix is an exponential and $\Lambda$ entries satisfy;

$$
\begin{array}{r}
\lambda_{-} i j \geq 0 \text { for } i \neq \mathrm{j} \\
\lambda_{i i}=-\sum_{j \neq i} \lambda_{i j}
\end{array}
$$

Equation 2 states that the elements in the diagonals have rows that sum to zero. The task remaining is in obtaining elements estimates of generator matrix $\Lambda$. The equation below gives the maximum likelihood estimate of $\lambda_{\mathrm{ij}}$.

$$
\widetilde{\lambda_{i j}}=\frac{N_{i j}(T)}{\int_{0}^{T} Y_{i}(s) d s}
$$

Where $N_{i j}(T)$ is the Total number of transitions in period $\mathrm{i}$ to $\mathrm{j}$ when $i \neq j$.

$Y_{i}(s)$ at time $s$, is the number of firms with rating $i$.

The number of years spent in istate is the denominator. Hence for one year, whether a firm spent part of it on transit e.g. from AA to A and ending the year with BBB that time portion spent in 'A' will have a contribution in the transition probability estimate $P_{A A \rightarrow A}$. This information is ignored in Cohort method. Even firms at period end have 'NR' status are counted in the denominator in the time portion spent in state $i$.

\subsection{Case of Non-Homogeneous}

Transitional data information is more efficiently used by duration approach than Cohort method. At the portfolio method or instrumental level, a common credit modeling assumption is that the system is first order Markov and this implication or assumption is very convenient.

The $k$-period Credit Transition matrix $P_{k \Delta t}$ is $P_{\Delta t}^{k}$ for a given period $\Delta \mathrm{t}$ e.g one quarter. For example you can compute a 1-Year transition matrix by simply raising to the $4{ }^{t h}$ power a 1 -Year transition Matrix. Non Markovian behavior e.g. non-homogeneity time which is sensitive to the business cycle and ratings drift have been shown by Bangla et al(2002), Altman and Kao(1992) and Carthy and Fons(1993). In reality, the migration matrix and the economy will change on a shorter than the required time scale to achieve the ideal Default Steady-state described by migration matrix which was assumed to be constant. From time $s$ to $t$, letting $P(s, t)$ be the transition probability matrix (Lando and Skodeberg (2002), the $\mathrm{i}^{t}{ }^{t h}$ element denotes the probability of Markov process moving from state $i$ at date $s$ to state $j$ at date $t$. O ne can estimate $P(s, t)$ consistently given $m$ transition sample over period $s$ to $t$ using Aalen-Johansen estimator (Aalen and Johansen (1978)).

$$
\tilde{P}(s, t)=\prod_{i=1}^{m}\left(I+\Delta \tilde{A}\left(T_{i}\right)\right)
$$

$T_{i}$ is the time interval jump from $s$ to $t, m$ is the transition days total relevant to the horizon i.e where there is at least one rating occurrence in the year. Also 


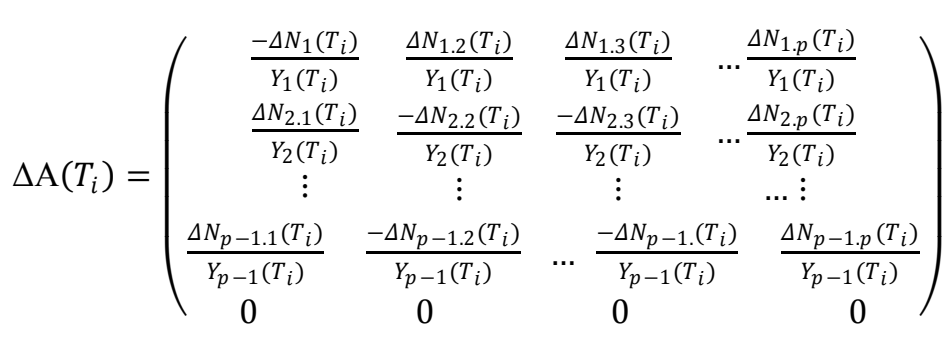

The Aalen-Johnsen estimator which is non parametric applied to intervals which are very short is the frequentist (cohort) method. The observation of the number of transitions from $k$ state to $j$ state is denoted by $\Delta N_{k, j}(T)_{i}$. The count of the Total transition number from $k$ state at date $T_{i}$ is denoted by diagonal elements $\Delta N_{k}\left(T_{i}\right)$ and the number of firms exposed or at risk is $Y_{k}\left(T_{i}\right)$. The exposed firms $Y_{k}\left(T_{i}\right)$ fraction leaving the the state at date $T_{i}$ is given by the count of row $k$ diagonal element $\Delta\left(T_{i}\right)$. Hence the terms of off diagonal transitions specific type normalized by the exposed firms number away from the state. The last row of $\Delta A\left(T_{i}\right)$ is zero as in the homogeneous case since default is a state absorbing and the rows of matrix $I+\Delta\left(T_{i}\right)$ add to one automatically. The Aalen-Johansen estimator which is nonparametric has the least assumptions on the process of generating data by taking into account the time homogeneity while all movements within the sample are fully provided for. However, it is not clear whether the least assumption of time homogeneity results in having different estimated migration matrices results which are different significantly.

\subsection{Matrices Comparison}

Three different methods of credit Migration Matrix entries estimation have been described above and the results need to be compared. Several ways of matrices comparison are available including L1 and L^2 distance matrices (Euclidean), eigen value and eigenvector analysis e.g singular value decomposition. Jaffry and Schuermann (2003) presented a discussion on Credit migration matrices metrics extensively and argued for a singular value metric which is commonly used.

\subsection{Identity matrix subtraction}

By definition, the migration matrix quantitatively determines how the migration of a given state vector from one epoch to the next epoch. Hence the main matrix characteristic is the migration amount from one epoch to the next imposed on the vector state and the highlight of this characteristic is by simply subtracting the identity matrix before commencing to other manipulations. There is a correspondence between the identity matrix and the static migration matrix i.e. from one epoch to the next, the state vector matrix remains unchanged. Subtracting the identity matrix from the credit migration matrix leaves one with the dynamic part only of the original matrix and devising this dynamic part leads to a result which reflects the matrix magnitude in implied mobility terms. The mobility matrix will be denoted $\mathrm{P}^{\sim}$ i.e it is original matrix minus the identity matrix and all should be of the same dimension i.e

$$
\widetilde{P}=P-I
$$

\subsection{Singular Values Metric}

Singular values of mobility matrix $\tilde{P}$ following Jaffry and Schuerman (2003) given by;

$$
m_{s v d}\left(\tilde{P}^{a}, \tilde{P}^{b}\right)=\overline{S\left(\tilde{P}^{a}\right)}-\overline{S\left(\bar{P}^{b}\right)}
$$

The overbar density vector $s(\tilde{P})$ average value, which also denotes the singular values of $P$ defined;

$$
S(P)=\sqrt{\operatorname{eig}\left(P^{I} P\right)}
$$

The singular matrix of $P$ are always positive while the eigen value of $P$ are sometimes negative hence to interpret distance measure becomes difficult. A viable metric is yielded as a result since average migration probability across all state is approximated.

\subsection{Bootstrap Technique}

Transition or Credit Migration matrices are noisy or error estimates of distance metrics $m_{s v d}\left(\tilde{P}^{a}, \tilde{P}^{b}\right)$. To know the size of distance metrics e.g., $m_{s v d}\left(\tilde{P}^{a}, \tilde{P}^{b}\right)$. Properties of $m_{s v d}\left(\tilde{P}^{a}, \tilde{P}^{b}\right)$ are needed. The $\mathrm{m}$ ost efficient and straight forward way in the absence of the estimates in the asymptotic properties of $m_{s v d}\left(\tilde{P}^{a}, \tilde{P}^{b}\right)$ is the bootstrapping technique. For example, if $\tilde{P}_{t}^{c}$ and $\tilde{P}_{t}^{b}$ are estimates of 
Cohort and Homogeneous methods respectively obtained by $N_{t}$ observations, then $N_{t}$ bootstrap samples are created in order to compute $k$ based differences values of singular nature;

$$
\left\{m_{\text {svd }}^{(j)}\left(\tilde{P}^{a}, \tilde{P}^{b}\right)\right\}_{j=1}^{k} \quad \text { where } j=1, \ldots, k
$$

denoting the replication number of bootstrap. This will result in distribution of bootstraps which are based on singular value distances. For example, take a value $\alpha=5 \%$ and find out whether 0 lies witjin the range of $1-\alpha$ of

$$
\left\{m_{s v d}^{(j)}\left(\tilde{P}^{a}, \tilde{P}^{b}\right)\right\}_{j=1}^{k}
$$

Running bootstrap technique for $N_{1999}$ firms sample and obtaining

$$
\left\{m_{s v d, 1999}^{(j)}\left(\tilde{P}^{a}, \widetilde{P}^{b}\right)\right\}_{j=1}^{k}
$$

for some large value $k \approx 1000$.

If $1-\alpha=95 \%$ results to $(-0.055,-0.005)$, then the null hypothesis is rejected and we state with $95 \%$ confidence that there is a significant difference between the two matrices with respect to the singular value based metric.

If the result is $(-0.055,0.005)$, then the null hypothesis is not rejected and we accept that the two matrices are identical with $95 \%$ confidence. Bootstrap technique requires that the data should be from a random sample. It is easier dealing with data from short horizons eg one year than with data across several years.

\section{Empirical results}

In this Section, the Frequentist Method (Cohort approach), Time Homogeneous and NonHomogeneous duration method will be compared using the $m_{s v d}$ metric on Credit Migration matrices estimates from one year Horizon common in many applications of Risk Management. It will be shown that differences between Time Homogeneous and Non Homogeneous duration methods are smaller than the differences between Frequentist Method (Cohort approach) and the duration methods. This implies that the Duration method is more efficient than the Frequentist method (Cohort approach).

Using rating histories secondary Data from U.S. obligors 1991-2011, we compute the $m_{s v d}$ of frequentist(Cohort approach) and subtract Homogeneous duration, again compute $m_{s v d}$ of Frequentist (Cohort approach) and subtract Non-Homogeneous duration and lastly compute the $m_{s v d}$ of Non-Homogeneous Cohort and subtract the Homogeneous Duration.

Making use of the SVD Metric, the Minima and Maxima results are summarized in the table below;

Table 1: Min and Max $\boldsymbol{m}_{\text {svd }}$. SVD migration Matrix estimated using Secondary Data S\&P U.S obligors 19912011.

\begin{tabular}{|l|l|l|}
\hline & Min(Year) & Max(Year) \\
\hline Frequentist(Cohort) minus Homogeneous Duration & $0.000732(1994)$ & $-0.05255(2009)$ \\
\hline Frequentist(Cohort) minus Non-Homogeneous Duration & $0.000355(1992)$ & $-0.06045(2009)$ \\
\hline Non-Homogeneous Duration minus Homogeneous Duration & $0.000077(2000)$ & $0.01005(1992)$ \\
\hline
\end{tabular}

The least difference is in the Year 1994 and the most difference is in 2009 between Frequentist (Cohort approach) and Homogeneous duration methods. In absolute value, the least occurs in 1994(0.000732) and the most in 2009(-0.05255).

The left column of Table 2 below illustrates the Bootstrap technique summary statistics which also includes quantiles.

Table 2: Bootstrap SVDs: $\boldsymbol{m}_{\text {svd }}\left(\widetilde{\boldsymbol{P}}^{\boldsymbol{c}}, \widetilde{\boldsymbol{P}}^{\boldsymbol{h}}\right), \boldsymbol{m}_{\boldsymbol{s v d}}\left(\widetilde{\boldsymbol{P}}^{n \boldsymbol{h}}, \widetilde{\boldsymbol{P}}^{\boldsymbol{h}}\right)$. Bootstrap Quantiles (k=1000). SVD distance metric using S\&P U.S Obligors, 1991-2011.

\begin{tabular}{|l|l|l|l|l|}
\hline & \multicolumn{2}{|l|}{$\begin{array}{l}\text { Frequentist(Cohort) minus } \\
\text { Homogeneous Duration }\end{array}$} & \multicolumn{2}{l|}{$\begin{array}{l}\text { Non-Homogeneous minus } \\
\text { Homogeneous Duration }\end{array}$} \\
\hline Stats & 1994(min) & 2009(Max) & $\mathbf{2 0 0 0 ( M i n )}$ & 1992(Max) \\
\hline$\widetilde{m}_{S V D}$ & 0.000732 & -0.05255 & 0.000077 & 0.01005 \\
\hline Mean & 0.0006 & -0.0509 & 0.000335 & 0.0110 \\
\hline st. dev & 0.0057 & 0.0162 & 0.001970 & 0.0107 \\
\hline Q1 & -0.0163 & -0.0905 & -0.00499 & -0.0015 \\
\hline Q5 & -0.0108 & -0.0765 & -0.00318 & 0.0009 \\
\hline
\end{tabular}




\begin{tabular}{|l|l|l|l|l|}
\hline Q50 & 0.0015 & -0.0509 & 0.00044 & 0.0082 \\
\hline Q95 & 0.0079 & -0.0241 & 0.00326 & 0.03251 \\
\hline Q99 & 0.0113 & -0.0129 & 0.005421 & 0.05151 \\
\hline
\end{tabular}

We are not able to reject that the Matrices of 1994 are different since 0 tends to be closer to the median but we can reject for Matrices of 2009. From $1^{\text {st }}$ percentile to $99^{\text {th }}$ percentile, the $98 \%$ confidence interval is ($0.0905,-0.0129)$ and 2009 density is wider than 2004 density since 2009 density is concentrated highly around zero. Comparing further the two methods of duration, the largest difference occurs in 1992(0.01005) followed closely by 2009(0.0080) and the smallest being in 2000(0.00008). 1994 was the year where we had the least difference between Frequentist (Cohort) method and and Homogeneous Duration and it was also small with the difference between methods of Duration. Table 2 above shows the result in the Right Hand Side column for Bootstrap for 1992 where we had the maximum difference and the minimum difference. We cannot reject the Hypothesis that there is zero difference even for the years having the maximum difference. It is clear that in terms of SVD, the difference between the methods of Duration are smaller significantly than the difference between the Frequentist (Cohort) and Methods of Duration implying that the methods of Duration are more efficient than the Frequentist (Cohort) method even with time-homogeneity assumption as illustrated in table 3 below.

Table 3: Methods differences in $\boldsymbol{m}_{s v d}$ Means: F-test makes an assumption of different variances. Each method $m_{\text {svd }}$ estimated from secondary data S\&P rated U.S Obligors 1991-2011.

\begin{tabular}{|l|l|l|l|l|}
\hline & $\begin{array}{l}\text { Freq(Cohort)-Homog } \\
\text { Duration }\end{array}$ & $\begin{array}{l}\text { Freq(Cohort)-Non Homog. } \\
\text { duration }\end{array}$ & $\begin{array}{l}\text { Freq(Cohort)- } \\
\text { Homog. Duration }\end{array}$ & $\begin{array}{l}\text { Non Homog. Duration } \\
\text { Hom }\end{array}$ \\
\hline Mean & -0.0123 & -0.0142 & -0.0123 & 0.0021 \\
\hline Std.Dev & 0.0194 & 0.0201 & 0.0191 & 0.0043 \\
\hline $\mathrm{F}(\Delta$ mean $=0)$ & \multicolumn{2}{|c|}{0.953} & \multicolumn{2}{c|}{} \\
\hline $\operatorname{Pr}(F \leq f)$ & 0.457 & & 29.718 \\
\hline
\end{tabular}

Means comparison between the different methods is done in Table 3 above. The difference between the Frequentist (Cohort) method and Homogeneous Duration is -0.0123 and the difference between the Frequentist (Cohort) and non homogeneous Duration method is -0.0142 . Lastly, the difference between the Nonhomogeneous duration method and Homogeneous Duration method is 0.0021 which is much smaller. Hence we cannot reject that the differences averages between frequentist (Cohort) method and the two duration methods with a value of p being 0.457 is different but we can reject for the difference between Frequentist (Cohort) and Homogeneous Duration method and the difference between the two methods of Duration with a p-value being 0.000012 . Obviously, the divergence degree is a time horizon function between the Frequentist(Cohort) and either of the Duration methods hence the migration is more potential with longer Horizon and the differences for shorter Horizons will be smaller e.g for quarterly or semi annually Horizons. Many applications of Credit nature follow one year horizon.

\section{Conclusion}

In this research paper, three methods of estimation namely Frequentist (Cohort), homogeneous Duration and Non-Homogeneous duration have been presented. Although frequentist (Cohort) estimation method is popular, it is inefficient. The two Duration methods namely Non Homogeneous and Homogeneous Duration are more efficient. To measure the scalar differences and asses whether the differences are statistically and economically significant in Credit Matrices, the focus first is made on the mobility matrix defined as $P-I$ where $P$ is the migration Matrix and $I$ is the identity Matrix; both $P$ and $I$ should be the same size. The static migration matrix corresponds to the identity Matrix . Subtracting the identity Matrix $I$ from the Migration Matrix $P$, we are left with dynamic part from the original matrix. Following Jaffry and Schuermann (2003) who developed Singular Value Decomposition SVD which is Metric based, we can argue that the singular values which are Metric based capture best the dynamic properties of the transitional or migration matrix. The differences are Statistically and economically important in that the two approaches estimate the Credit risk level of Capital by simulating estimates used for generators of Credit assets portfolios e.g Bonds and Loans and then using Kijima and Komoribayashi(1998) pricing model, the Credit derivative is priced in what is known as Credit yield spread. Statistically and economically, the method is useful in analysis of estimated Credit migration matrices usually for 1 year period horizon common in most applications of Risk management nature, In cases where the SVD netric is sufficiently small, the null hypothesis that they are not different cannot be rejected but we can reject the null hypothesis in cases where the SVD metric is sufficiently large. From the Credit Risk Capital resulting from Credit portfolio model, the difference between the Frequentist(Cohort) method and the Duration models which are more efficient is bigger than the difference between the Duration methods. The differences for the former range from $30 \%$ to almost $50 \%$ but for the latter, the difference is not more than $2 \%$. The differences noted are also replicated in Risk Capital which also affect the Business Cycle by 
almost $40 \%$ hence if one uses the wrong Matrix in Credit derivative prices, it can result in mispricing by almost $50 \%$ which is a very big margin.

\section{References}

[1] Aalen O.O and S Johansen 1978"An Empirical Transition matrix for non-homogeneous

[2] Markov chains based on Censored observations". Scandinavian journal of Statistics.

[3] Altman E.I and D.L kao 1992, "Rating Drift of High Yield Bonds"3. Arvinitis, A,J Gregory and J-P Laurent 1999, "Building Models for Credit Spreads. journal of Banking and finance.

[4] B. Belkin, S. Suchower and L.Forest Jr. (1998), "A one parameter presentation of Credit Risk and Transitional Matrices"

[5] Bahar R. Nagpal. K, 2000, "Rating Transitions: Modelling the dynamics",

[6] Cantor R and F Packer, 1995 "The Credit Rating Industry"

[7] Christensen, J and D. Lando, 2002, "Confidence sets for continuous time rating tran- sition probabilities"

[8] Geweke J, R.C Marshall and G.A Zarkin 1986, "Mobility indices in continuous Time Markov chains".

[9] Israel, Robert B, Jeffrey S. Rosenthal and Jason Z Wei, 2001, "Finding Generators for

[10] Markov chains via empirical Transition Matrices with Application to credit Ratings"

[11] Jaffry Y and T.Schuermann, 2003, "Metrics for comparing Credit Migration Matrices"

[12] Jarrow R. A, D. Lando and S.M Turnbull 1997, "A Markov Model for the Term Struc-ture of Credit Risk Spreads". 